\title{
A computerized Langmuir probe system
}

\author{
L. S. Pilling, ${ }^{\text {a) }}$ E. L. Bydder, and D. A. Carnegie \\ Department of Physics and Electronic Engineering, University of Waikato, Hamilton, New Zealand 2001
}

(Received 27 December 2002; accepted 2 April 2003)

\begin{abstract}
For low pressure plasmas it is important to record entire single or double Langmuir probe characteristics accurately. For plasmas with a depleted high energy tail, the accuracy of the recorded ion current plays a critical role in determining the electron temperature. Even for high density Maxwellian distributions, it is necessary to accurately model the ion current to obtain the correct electron density. Since the electron and ion current saturation values are, at best, orders of magnitude apart, a single current sensing resistor cannot provide the required resolution to accurately record these values. We present an automated, personal computer based data acquisition system for the determination of fundamental plasma properties in low pressure plasmas. The system is designed for single and double Langmuir probes, whose characteristics can be recorded over a bias voltage range of $\pm 70 \mathrm{~V}$ with 12 bit resolution. The current flowing through the probes can be recorded within the range of $5 \mathrm{nA}-100 \mathrm{~mA}$. The use of a transimpedance amplifier for current sensing eliminates the requirement for traditional current sensing resistors and hence the need to correct the raw data. The large current recording range is realized through the use of a real time gain switching system in the negative feedback loop of the transimpedance amplifier. (C) 2003 American Institute of Physics. [DOI: 10.1063/1.1581362]
\end{abstract}

\section{INTRODUCTION}

Langmuir probes are an invaluable tool for plasma diagnostics. Through the use of different probe configurations they allow the determination of not only densities and temperatures, but also provide detailed information on the type and nature of the distribution functions of the charged species. ${ }^{1}$ The latter measurements are important since these data are almost impossible to obtain from any other measurement technique. Since the Maxwell-Boltzmann distribution is a theoretical limit, only capable of being achieved if the entire plasma and its surroundings are in complete thermodynamic equilibrium, some deviation from the theoretical curves is to be expected in any laboratory plasma. This deviation usually manifests itself in the loss of the high energy electrons from the tail of the distribution.

For non-Maxwellian plasma distributions of the charged species, one probe technique alone is often not sufficient to determine the nature of the electron and ion distribution functions. ${ }^{2,3}$ Specifically for those plasmas with nonMaxwellian distribution functions, single probe analysis will, in general, show how many different electron distributions are present. This analysis will provide accurate determination of the lower temperature distributions but for the highest energy distribution the measured temperature can be, and often is, inaccurate. ${ }^{4}$ This inaccuracy arises since the electron current becomes increasingly sensitive to the removal of the ion saturation current as the high energy tail of the distribution function becomes more depleted. That is, the determination of the electron temperature becomes increasing inaccu-

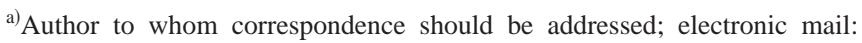
lsp@phys.waikato.ac.nz
}

rate as the electron energy distribution function moves from a Maxwellian type distribution towards a mono-energetic distribution. It is therefore important to measure the ion saturation current as accurately as possible to minimize the errors in the high temperature determination in these types of plasmas. Even for high density plasmas with clearly Maxwellian distributions, it is becoming apparent that high accuracy recording of the ion saturation curve is necessary to model the ion current to obtain self consistent electron densities. ${ }^{5}$

One of the fundamental problems with probe analysis is that it is not known a priori which probe theory will apply since the state of the plasma is unknown. Thus to best determine the plasma properties, it is prudent to obtain both single and double probe data from the same plasma conditions at the same time. The measurements should be carried out over the widest possible current range including the saturation regimes of the ions and electrons in order to be able to apply all theoretical models. This leads to difficulties in the external Langmuir probe recording circuitry since the currents recorded from the different probe configurations are orders of magnitude apart and cannot be recorded with a single current sensing resistor to obtain the accuracy needed.

In order to obtain these data, an electronic circuit configuration was devised to allow the simultaneous recording of these widely disparate values from each probe configuration. The circuits were designed with the following goals in mind: First to record the correct voltage and current drawn by the probe to avoid data correction or manipulation, ${ }^{6}$ and second to allow the range of current recording to be as large as possible without compromising accuracy of the recorded data. We will first briefly review previous circuit designs, and then present our circuit description. 


\section{Floating Resistor}

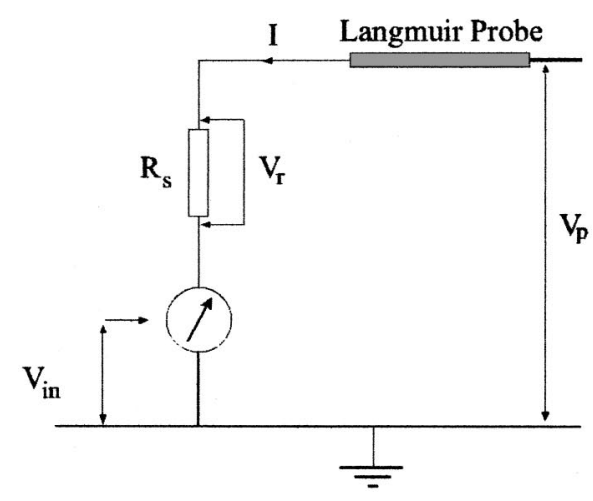

Floating Power Supply

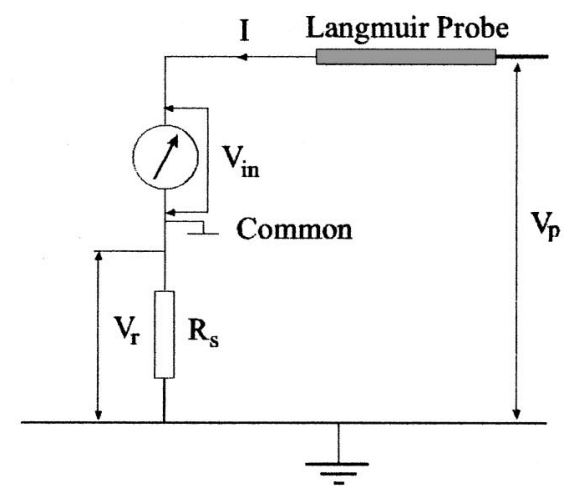

FIG. 1. Generalized circuit descriptions where either the sensing resistor or the power supply is floating.

\section{ESTABLISHED DATA ACQUISITION SYSTEMS}

Early attempts to digitally acquire Langmuir probe data from plasmas were expensive and time consuming and remained so until personal computers became widely available. One of the first automated systems was constructed by Hopkins et al. ${ }^{7}$ who described what was at the time a remarkably complex and advanced Langmuir probe acquisition and analysis system. The probe was controlled by a $\pm 30 \mathrm{~V}$ sweep over 1000 points. This sweep could be offset from 0 to $-150 \mathrm{~V}$. The data acquisition system chose from multiple resistors for the best current resolution and calculated the plasma parameters such as density and electron temperature automatically at the end of acquisition.

Later authors used acquisition systems such as simple boxcar averaging to an oscilloscope as employed by Shindo et $a l^{8}{ }^{8}$ or the more elaborate configurations of Sudit and Woods. ${ }^{6}$ The majority of these methods used a current sensing resistor in series with the Langmuir probe. This is the simplest method of recording the voltage across, and current drawn to, the probe. Although simple to implement, there are certain problems with this approach. The first is whether to float the sensing resistor and ground the power supply ${ }^{9-12}$ or conversely, to float the power supply and ground one side of the sensing resistor. ${ }^{7,13-17}$ These two configurations are shown pictorially in Fig. 1. Both methods have drawbacks noted by Duffey et al. ${ }^{16}$ Detection of the differential voltage signal is difficult since neither side of the sensing resistor is grounded in the first case. In the second case, floating the power supply adds complications to the circuit design as well as obscuring the relationship between the voltage from the supply and the probe potential. Another difficulty, for both configurations, is the need to correct for the potential developed across the current sensing resistor to obtain the true voltage applied to the probe. ${ }^{6}$ This correction is necessary to ensure the actual voltage applied to the probe is recorded, and secondly to evenly space the voltage data. The latter simplifies smoothing the data and obtaining the derivatives of the probe characteristics if the numerical methods of Savitsky and Golay ${ }^{18}$ are to be used. For all the complexities of these systems, they still need to be corrected for the voltage drop over the current sensing resistor and they all can only acquire the full probe characteristic using a single resistor, or gain setting at a time.

A further problem occurs with the use of a single sensing resistor to record the current to the probe. A Langmuir probe data acquisition system is typically designed so that a maximum of $200 \mathrm{~mA}( \pm 100 \mathrm{~mA})$ can be drawn to the probe. Generally the analog to digital converter (ADC) and digital to analog converter (DAC) have 12 bit resolution. For this current range, the minimum detectable signal [the least significant bit (LSB)] is $48.8 \mu \mathrm{A}$. For recording accuracy, a minimum of 50 of these steps is necessary to record a smooth data curve. This implies that the recorded current should not fall below $2.44 \mathrm{~mA}$. This is the minimum level the ion current should be and falling below this value degrades the signal into a stepwise function, limiting accuracy of the ion current which is needed to calculate the plasma parameters. Therefore, to obtain smooth data curves with a single resistor over in the submicron current range requires DAC/ADC converters with at least 24 bits. A better and less expensive solution is achieved by introducing multiple sensing resistors, as we will show in Sec. III.

\section{PHYSICAL AND ELECTRONIC CONSTRUCTION}

The experimental plasma discharge we used to acquire Langmuir probe data was a thermionically enhanced dc discharge plasma. Details of the system can be seen in Fig. 2. The system has a base pressure of $10^{-6} \mathrm{mbar}$ while Langmuir probe experiments were conducted at pressures of $10^{-2}$ mbar in a nitrogen atmosphere. This experimental arrangement produced a stable plasma discharge that was free from interference and magnetic fields to test the Langmuir probe circuitry. We wished to avoid complications caused by the rf fluctuations in the plasma and the modifications to the data acquisition circuitry necessary for the probe to follow the rf field. We also wanted a plasma where a two temperature electron distribution would thoroughly test the circuitry and the subsequent probe analysis.

Standard operation of the apparatus was to set the dc bias to approximately $300 \mathrm{~V}$ and to slowly increase the filament current until the electron emission allowed a plasma to form. For this arrangement, the conditions necessary to initiate a plasma were more constrained than the stable plasma discharge range. Once the plasma was established, the filament current and the dc bias were adjusted to provide a 




FIG. 2. The experimental plasma chamber layout.

stable plasma within the range of $100-300 \mathrm{~V}$ dc to allow plasma currents of $0.5-2.5 \mathrm{~A}$.

After reviewing previous circuit descriptions, we decided to build a completely automated control system incorporating the features we wanted for the recording of data from the Langmuir probes. Sophisticated electronics had to be designed, constructed, and tested before experimental data could be obtained. The specifications for the design were based on simulation voltage-current data obtained by inserting the expected temperature and density ranges into the standard Langmuir probe theories. ${ }^{2,3}$ From this study, it was determined that the voltage applied to the probe would need to be $\pm 70 \mathrm{~V}$ while the current range required measurement from nanoamperes to milliamperes in order to measure, with full resolution, the ion and electron saturation currents drawn to the single and double probes.

The Langmuir probes used in the simulation and in practice were $1 \mathrm{~cm}$ long with a radius of $0.1 \mathrm{~mm}$. This gives an aspect ratio of 100 allowing the end effects of the probe to be ignored. Tungsten wire was used as the probe material where $1 \mathrm{~cm}$ was protruding from an alumina tube which itself was enclosed in a glass tube.

Figure 3 illustrates the main components of our circuit while Fig. 4 shows the generalized layout compared to previous designs (see Fig. 2). The use of a transimpedance amplifier allows the Langmuir probe current to be measured without affecting the voltage applied to the probe and eliminates the need for a conventional current sensing resistor. This design isolates the current sensing resistors from the

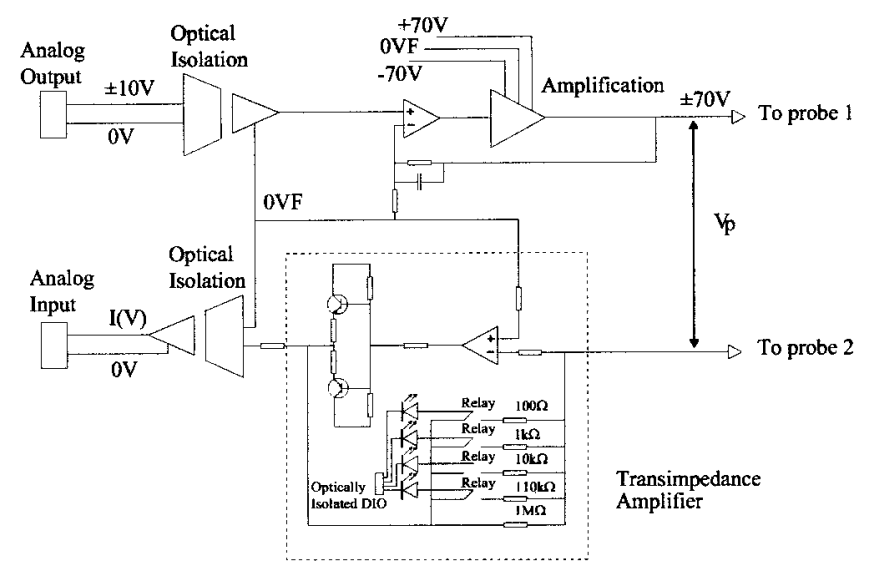

FIG. 3. A simplified circuit diagram used to acquire data from the Langmuir probes.

probe voltage as the feedback loop ensures both the inputs to the operational amplifier are at the same potential so that the voltage across the probe is the same as the applied voltage from the electronic circuits. The current sensing resistors are therefore effectively removed from the probe system allowing us to float the power supply to obtain an entirely floating system referenced to a virtual ground. The resistor values were chosen to be $1 \mathrm{M} \Omega-100 \Omega$ changing by factors of 10 over five current ranges (see Fig. 3). This gave us a current sensing range of $200 \mathrm{~mA}$ ( $\pm 10 \mathrm{~V}$ over the sensing resistors) with a LSB of $4.88 \mathrm{nA}$, allowing a minimum safe current detection of $244 \mathrm{nA}$ assuming an accuracy of 50 steps.

The operational control was based on a personal computer running Windows NT 4.0 utilizing a National Instruments model MIO-16H9 data acquisition board and LABVIEW software. To ensure that the recorded data was spaced in time correctly, the onboard Am9513A frequency clock was used to trigger the DAC. On this type of data acquisition board (DAQ), a square wave signal is able to be referenced using the frequency out pin (FOUT). The Am9513 clock frequency can be chosen from five ranges $1 \mathrm{MHz}, 100 \mathrm{kHz}, 10 \mathrm{kHz}, 1$ $\mathrm{kHz}$, and $100 \mathrm{~Hz}$ and there is a divisor for each range varying from 1 to 16 providing a set frequency, or sample acquisition rate of choice. For example, with the $10 \mathrm{kHz}$ clock selected, the frequency choices range from $10 \mathrm{kHz}$ to $625 \mathrm{~Hz}$ (divisors 1 and 16). The clock output can be connected directly to the DAC update pin on the data acquisition board

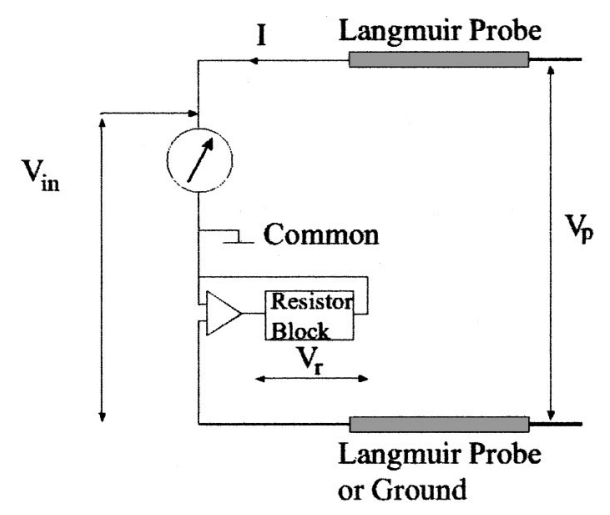

FIG. 4. The generalized operational form of the entire Langmuir probe circuitry. 
allowing consistent external timing control of the sampling rate as well as evenly spacing the data in time. This removes operating system software problems and hardware latencies inherent in most computer systems. ${ }^{19}$ The full circuit design is based on two circuit boards. A general control board splits the 50 pin cable from the DAQ board into its separate components, i.e., analog input and output, digital input and output (DIO). Specific lines are then connected to the probe driver circuit shown in Fig. 3. Each Langmuir probe circuit board requires one analog input and output line and four DIO lines for control of the transimpedance gain resistors. In total, one general control board and two Langmuir probe boards can be simultaneously driven by the system allowing data acquisition from two independent probe systems.

The programs written to control the acquisition were written in LABVIEW and could be operated in three ways:

(1) slew mode, using a continuously repeated ramp voltage between the high and low voltage values to acquire any number of probe traces; or

(2) single shot, where any portion of the characteristic can be recorded by adjusting the voltage sweep range over an arbitrary number of points; and

(3) using a triangle wave as the voltage source to check the probes for hysterisis.

However any signal that can be digitized or described by a function can be used as the voltage wave form. If one could determine a theoretical single probe response to a known voltage range, ${ }^{5}$ or determine how $V(r)$ varied with $I_{p}$ from the theories of Chen ${ }^{20}$ or Laframboise, ${ }^{21}$ it should be possible to translate this into the input voltage signal function for which the current recorded would be linear. For example, if the ion current obeys the orbital motion limited theory then $I_{p} \propto V^{1 / 2}$. Thus if a ramp voltage of $V^{1 / 2}$ instead of a linear ramp voltage was applied to the probe, the current response should be a straight line with a slope of -1 where the theory holds.

Normal operation of the system includes the use of the gain switching system. The $\pm 10 \mathrm{~V}$ from the DAQ card is converted to a $\pm 70 \mathrm{~V}$ output to the Langmuir probe. The current from the probe is recorded for each voltage step at a sampling rate of up to $75 \mathrm{~Hz}$. During acquisition, the voltage across the gain resistors is checked as each point is recorded to ensure that the voltage is within the operational recording range. If the voltage is out of the range assigned to the gain settings, automatic switching occurs to a more appropriate gain resistor for the next sample. This datum point is still valid and does not need rerecording at the new gain setting. The voltage levels at which the gain switches allows for the previous point to be recorded correctly. These levels are usually set at \pm 0.4 and $\pm 7 \mathrm{~V}( \pm 10 \mathrm{~V}$ over the sensing resistors). For instance, if the voltage across the gain resistor is positive and rising, once it passes $7 \mathrm{~V}$ the next higher gain resistor is switched to where the voltage is now $0.7 \mathrm{~V}$ but the recorded current is identical on both gain resistors. This switching of gain resistors occurs for all four possible choices. These limits depend on the instantaneous change in the current to the probe with changing voltage. This is pre- dominantly through the electron current collection regime. As long as the previous point does not saturate the $\pm 10 \mathrm{~V}$ input of the DAQ card, there is no invalid data. This feature is especially useful when single probes are used as it permits the same resolution when recording the ion and electron currents.

At the present time, the gain switching causes the sample acquisition rate to be set very low. The limiting factor is the time needed for the mechanical reed relays to turn on or off upon switching the DIO lines which control the gain settings. These relays were chosen for their robustness but this came at the expense of speed. However these relays can be replaced with much faster digital equivalents which would allow real time gain switching acquisition at speeds close to the throughput of the DAQ board, 100 ksamples/s. Without the gain switching portion of the program the system can acquire data at speeds close to the theoretical sampling rate. Using LABVIEW as the programming language gives extensive flexibility in control of the circuitry and easily allows automatic post processing of the acquired data. The dc plasma system used for testing is stable and quiescent so the slow speed is of less concern than it would be in other plasmas with different generation methods.

\section{RESULTS AND DISCUSSION}

The electronic probe acquisition system was fully calibrated and tested using resistors of various magnitudes as well as diodes to tune the precision resistors, check the linearity of the entire electronics, and calibrate the gain switching system. In the circuit of Fig. 3 the lowest four resistors are switched in and out of parallel with the $1 \mathrm{M} \Omega$ resistor. This means that to achieve "equivalent" sensing resistors with the values $100 \mathrm{k} \Omega, 10 \mathrm{k} \Omega, 1 \mathrm{k} \Omega$, and $100 \Omega$ we require actual values of $111 \mathrm{k} \Omega, 10.1 \mathrm{k} \Omega, 1 \mathrm{k} \Omega$, and $100 \Omega$.

Each component in the feedback loop consists of a precisely known resistor value and a fine tuning precision resistor used to adjust the resistance of the feedback loop to the correct value. This allows for correction of the individual resistance values of the circuit. Furthermore, each gain setting, from the lowest to the highest $(\mathrm{nA}-\mathrm{mA})$ is tuned in sequence so that switching gains produce an identical result (e.g., $7.818 \mathrm{~V}$ becomes $0.7818 \mathrm{~V}$ ) on switching to the next highest gain level. This process is also implemented in reverse by switching to lower gain levels.

Once this process was completed satisfactorily, several resistors were chosen at random and their characteristics recorded by this system. The resistances were calculated using the slope of the current-voltage $(I-V)$ data and compared to a value obtained using a four-point impedance meter. The results were values correct to within $0.1 \%$ of the resistance value. For example, a $10.02 \mathrm{M} \Omega$ resistor, which switched across the lowest two gain settings, was calculated to have a resistance value of $10.03 \mathrm{M} \Omega$ using regression analysis of the raw $I-V$ data. No data smoothing, or averaging of successive scans was necessary.

To test the gain, resistors with specific values that would give an overlapping response of two gains were chosen for initial comparison. Once this was completed, recording across a diode in series with a resistor allowed a full test of 


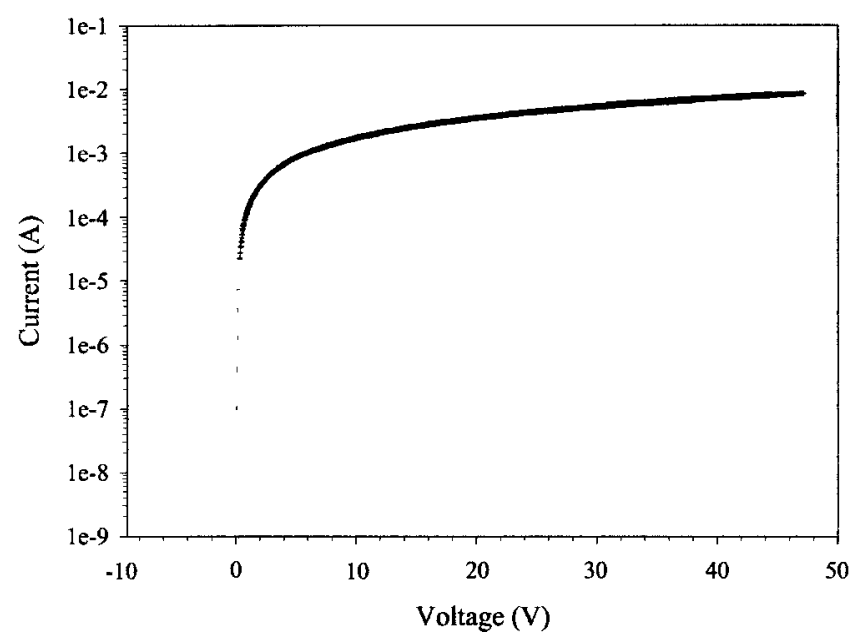

FIG. 5. Current passing through a diode showing the measuring range of the Langmuir probe circuitry.

the five gain bands through negative to positive voltages, a total of ten range changes. These raw $I-V$ data are presented in Fig. 5 on a semilog axis for clarity. In the calibration of the final design, the accuracy and precision of the system was found to be only limited by background noise, digitization errors, and nonlinearity inherent in the components used in building the system. This allows a detection accuracy greater than $0.2 \%$ over all gain settings for the linear and nonlinear resistive loads tested.

To test the system in a plasma we acquired multiple probe traces from a single set of plasma conditions. These were chosen to be a $300 \mathrm{~V}$ dc bias with the filament current adjusted to provide a plasma current of $1 \mathrm{~A}$ at a pressure of $5 \times 10^{-2}$ mbar. A typical smoothed single probe $I-V$ characteristic using the least squares method ${ }^{18}$ with a five point bin width is shown in Fig. 6. If we follow the general procedure $^{1-3}$ to obtain the electron current from these data, we calculate the ion current and subtract it from the total current to obtain the electron component and replot the data on a semilog axis as shown in Fig. 7. The electron temperature(s) can be obtained from the gradient(s) of the curves below the saturation region. We can see that two distributions are present for which the gradients provide tempera-

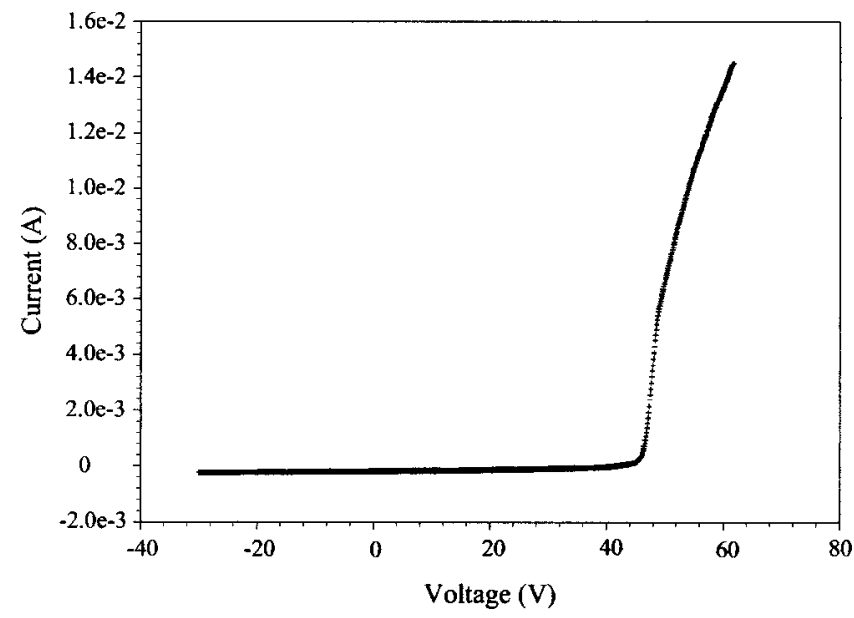

FIG. 6. A typical single probe characteristic.

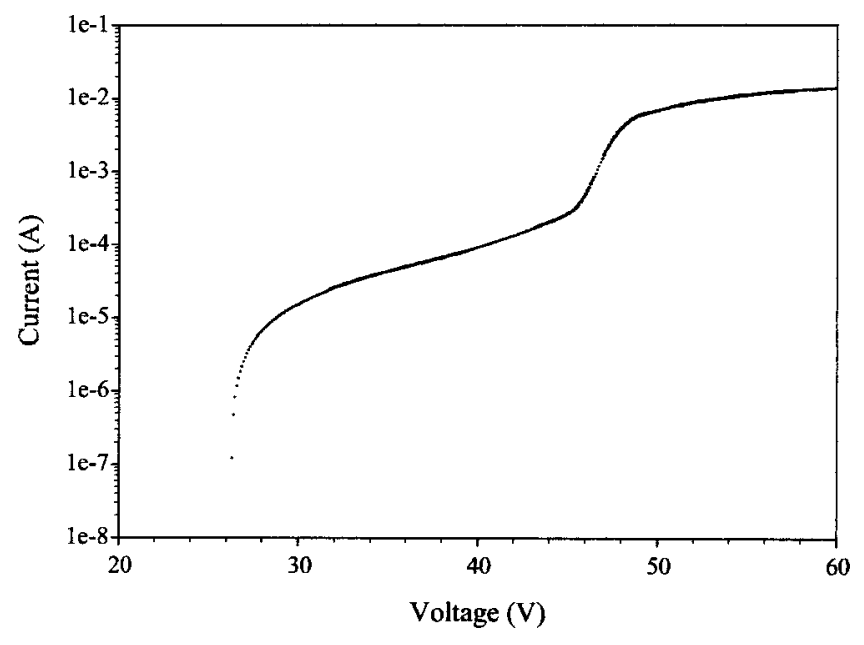

FIG. 7. A semilog plot of the $I-V$ characteristic showing a two temperature distribution.

tures of 6.67 and $0.9 \mathrm{eV}$. By looking at Fig. 6 it can be seen that the high energy tail appears depleted since the electron current contribution to the total current is collected over a narrow voltage range.

Due to this dual temperature distribution, data was obtained from a configuration of double probes to confirm the high temperature values. The arrangement of a double probe in its simplest form, occurs when the grounded side of the sensing resistor is connected to another single probe, as shown in Fig. 4. To operate a double probe system, the entire electronics system and power supplies must be able to float at the plasma potential and hence are insulated from ground. A double probe is always limited to drawing the ion current on one of the electrodes and by balance, sufficient electron current to balance the ion current to the other electrode. Thus they cannot sample the entire electron distribution but are limited to collecting electrons from the high energy tail of the distribution. For further details the reader is referred to the original paper of Johnson and Malter. ${ }^{22}$

If we look at the experimental double probe $I-V$ curves, we find the general form is that shown in Fig. 8. The temperature, which is $3.8 \mathrm{eV}$, can be calculated from knowledge of the saturation ion currents and the slope of the curve at the

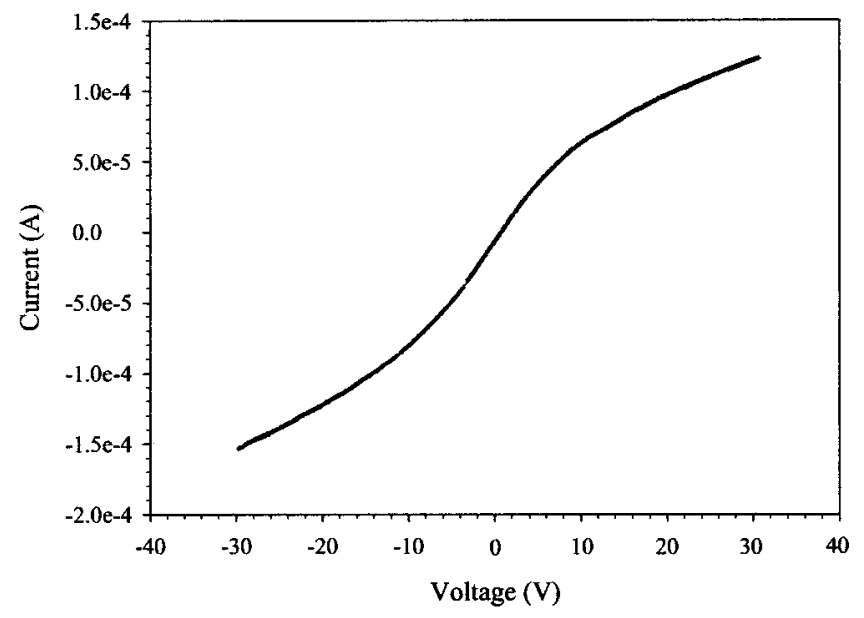

FIG. 8. A typical double probe characteristic. 
TABLE I. Comparative electron temperature values from single and double Langmuir probes.

\begin{tabular}{lccc}
\hline \hline & \multicolumn{2}{c}{ Single probe } & \\
\cline { 2 - 3 } $\begin{array}{l}\text { Data } \\
\text { set }\end{array}$ & $\begin{array}{c}\text { High temperature } \\
(\mathrm{eV})\end{array}$ & $\begin{array}{c}\text { Low temperature } \\
(\mathrm{eV})\end{array}$ & $\begin{array}{c}\text { Double probe } \\
\text { temperature }(\mathrm{eV})\end{array}$ \\
\hline 1 & 6.67 & 0.898 & 3.81 \\
2 & 5.69 & 0.898 & 3.76 \\
3 & 6.67 & 0.840 & 3.70 \\
4 & 7.57 & 0.853 & 3.88 \\
5 & 5.41 & 0.868 & 3.53 \\
6 & 5.98 & 0.860 & 3.62 \\
\hline \hline
\end{tabular}

origin. ${ }^{2,3}$ This temperature should be the same as the temperature from the high energy distribution of the single probe data but it compares badly being approximately half its value. If we repeat this analysis on multiple single and double $I-V$ curves taken under the same conditions we find that the temperature values from the high energy single probe distribution vary markedly, whereas the values from the low energy distribution and the double probe data are quite regular. These data are presented in Table I and shows that the double probe temperatures are more reliable and the high energy single probe temperatures are deemed to be erroneous. This conclusion comes from error analysis of the experimental data where it is found that a small variation in the ion current gives rise to a large variation in the apparent temperature obtained in a semilog plot method for a dual temperature distribution $^{23}$ (see Fig. 7). Since the temperature values obtained from single probe data are commonly used to calculate the density of the plasma, the errors in the temperature are translated through to this value making it inaccurate as well.
${ }^{1}$ J. D. Swift and M. J. R. Schwar, in Electrical Probes for Plasma Diagnostics (Iliffe Books, London, 1970).

${ }^{2}$ F. F. Chen, in Plasma Diagnostics Techniques, edited by R. H. Huddlestone and S. S. Leonard (Academic, New York, 1965).

${ }^{3}$ L. Schott, in Plasma Diagnostics, edited by W. Lochte-Holtgreven (NorthHolland, Amsterdam, 1968).

${ }^{4}$ V. A. Godyak, R. B. Piejak, and B. M. Alexandrovich, J. Appl. Phys. 73, 3657 (1993).

${ }^{5}$ F. F. Chen, Phys. Plasmas 8, 3029 (2001).

${ }^{6}$ I. D. Sudit and R. C. Woods, Rev. Sci. Instrum. 64, 2440 (1993).

${ }^{7}$ M. B. Hopkins, W. G. Graham, and T. J. Griffin, Rev. Sci. Instrum. 58, 475 (1987).

${ }^{8}$ H. Shindo, M. Konishi, and T. Tamaru, Rev. Sci. Instrum. 59, 2002 (1988).

${ }^{9}$ F. Fujita and H. Yamazaki, Jpn. J. Appl. Phys., Part 1 27, 1477 (1988).

${ }^{10}$ D. Fang, R. R. Williams, and R. K. Marcus, J. Anal. At. Spectrom. 5, 569 (1990).

${ }^{11}$ P. Spatenka, R. Studeny, and H. Suhr, Meas. Sci. Technol. 3, 704 (1992).

${ }^{12}$ G. Neumann, M. Kammeyer, and B. Schneemann, Meas. Sci. Technol. 6, 391 (1995).

${ }^{13}$ J. B. Friedmann, C. Ritter, S. Bisgaard, and J. L. Shoet, J. Vac. Sci. Technol. A 11, 1145 (1993).

${ }^{14}$ T. Kimura and K. Ohe, Rev. Sci. Instrum. 64, 3274 (1993).

${ }^{15}$ K. Hansen, T. Klinger, and A. Piel, Rev. Sci. Instrum. 65, 2615 (1994).

${ }^{16}$ B. Duffey, A. Cheetham, and J. Rayner, J. Electr. Electron. Eng., Aust. 16, 91 (1996)

${ }^{17}$ A. D. Cheetham, L. Davidson, J. Jakobsen, T. Lund, and J. Rayner, Rev. Sci. Instrum. 68, 3405 (1997).

${ }^{18}$ A. Savitsky and M. J. E. Golay, Anal. Chem. 36, 1627 (1964).

${ }^{19}$ G. B. Patel, P. K. Srivastava, L. M. Awasthi, V. P. Anitha, G. Ravi, P. J. Patel, U. K. Baruah, and S. K. Mattoo, Rev. Sci. Instrum. 73, 1779 (2002).

${ }^{20}$ F. F. Chen, J. Nucl. Energy, Part C 7, 47 (1965).

${ }^{21}$ J. G. Laframboise, in Rarefied Gas Dynamics, edited by J. H. De Leeuw (Academic, New York, 1966), Vol. II; National Technical Service Document No. AD634596 and University of Toronto Institute of Aerospace Studies Rep. No. 100.

${ }^{22}$ E. O. Johnson and L. Malter, Phys. Rev. 80, 58 (1950).

${ }^{23}$ L. S. Pilling, D. A. Carnegie, and E. L. Bydder (unpublished). 